\title{
Asymmetry in chromosome pairing: a major factor in de novo mutation and the production of genetic disease in man
}

\author{
ANN C CHANDLEY \\ From the MRC Human Genetics Unit, Western General Hospital, Edinburgh EH4 $2 X U$.
}

SUMMARY At the outset of the meiotic pairing process in man, trial and error mismatching and misalignment, both within homologous pairs and between heterologues, can be observed cytologically. Pairing starts at early zygotene principally within subtelomeric regions where the synaptonemal complex initiates. In the present paper, evidence for the primary role in synaptic initiation of a GC rich minisatellite in the human XY pseudoautosomal segment is presented, and circumstantial evidence is provided to support the view that GC rich sequences (minisatellites and Alu repeats) function to promote pairing within autosomes. The known sequence hypervariability of proterminal human minisatellites, it is suggested, arises as a secondary consequence of unequal exchange after misalignment between tandem repeats at the outset of the pairing process. Unequal exchange within misaligned repeat sequences at early prophase of meiosis could make a major contribution to de novo germinal mutation (conversion, duplication, deficiency, inversion, translocation), with serious consequences in man for the production of hereditary disease. For somatic tissues, rare mispairing between $\mathrm{G}$ rich repeats followed by unequal exchange could be a key step in cancer progression. It might also explain somatic mosaicism in some non-neoplastic clinical conditions.

A close approximation of homologous chromosome regions is an essential prerequisite for the exchange of genetic material. Yet, despite decades of cytogenetic investigation, ${ }^{1}$ the basic underlying mechanism by which eukaryote chromosomes achieve full homologous synapsis at meiotic prophase has so far eluded discovery. A principal impediment in the past has been lack of knowledge at the molecular level, a situation which has now changed quite remarkably. Among the many discoveries made by molecular biologists in recent years has been the finding that numerous families of repetitive DNA sequences are dispersed around eukaryote genomes. ${ }^{2}$ Loci with variable numbers of tandem repeats, VNTRs or 'minisatellites', ${ }^{3-6}$ have proved the most useful in genetic analysis, the repeat lengths being so variable from one person to another that they provide a source of DNA fingerprinting. ${ }^{34}$ Most minisatellites are GC rich $^{3-6}$ and show a strong tendency to cluster within proterminal regions (terminal $\mathrm{R}$ bands) of human chromosomes, ${ }^{6-8}$ including within the $X Y$ pseudoautosomal

Received for publication 2 March 1989.

Revised version accepted for publication 5 May 1989. region, ${ }^{910}$ and these are also regions of high recombinational proficiency. ${ }^{11} 12$ The occurrence of minisatellites across a range of eukaryotes, including plants, ${ }^{13}{ }^{14}$ suggests a conserved function, but this has not been precisely identified. Nevertheless, owing to features such as the $G$ richness of their common core sequence, which closely resembles the chi sequence in $E$ coli, Jeffreys et $a l^{34}$ have suggested that proterminal minisatellites might be recombinational signals in man.

A second important discovery has been that $\mathbf{R}$ and $G$ (dark) bands in the human genome are each characterised by distinct families of short interspersed repeat sequences, the SINES, Alu family dominating in $R$ bands, and the LINES, L1 family dominating in $G$ bands. ${ }^{15}$ Alu repeats are $G C$ rich and $L 1$ repeats are AT rich, but, again, no function has been ascribed to these repeats.

In the present paper, I wish to present data and supporting evidence for my belief that a prime function of GC rich repeated sequences in eukaryotyes is the initiation of pairing at meiosis. In man, the extreme variability of proterminal GC rich tandem repeats (VNTRs) ${ }^{3-6}$ arises, I believe, out of their 
synaptic role, unequal crossing over occurring within misaligned repeat arrays at early zygotene when a search for homology is first being made. Interstitial GC rich minisatellites and Alu-like sequences may also function in pairing initiation, the latter perhaps providing homology for pairing within gene loci. Asymmetrical alignment during synaptic trial events could, by unequal exchange,$^{16}$ generate an infinite variety of mutations from minor allelic changes (conversions) to structural rearrangements, for example, deletions, duplications, inversions, and translocations, contributing to genetic disease in man. ${ }^{17} 18$ The model has implications in cancer progression, since chance mispairings and unequal exchanges within GC rich sequences of somatic cells could also generate mutational change. ${ }^{1920}$ They might also form the basis of somatic mosaicism in some non-neoplastic conditions. ${ }^{21}$

\section{Evidence for the function of a human GC rich minisatellite in meiotic pairing initiation}

The first clue that a human GC rich minisatellite might initiate chromosome pairing came from a study into the meiotic pairing behaviour of the $X$ and $\mathrm{Y}$ axes in prophase spermatocytes of an STS deficient man showing a cytologically visible deletion at Xpter in his somatic karyotype (Mohandas et al, in preparation). Molecular analysis showed that all of the Xp pseudoautosomal region, as well as the MIC2 and STS genes, were deleted. At early zygotene, when XY pairing between the terminal regions of the two short arms normally starts in man, ${ }^{22}$ no pairing initiation was seen in this rare patient, despite the fact that the $X p$ and $Y p$ telomeres lay in close proximity in about one half of all cells examined at this stage. This observation indicated that telomeric recognition was, in itself, insufficient for proterminal pairing initiation and synaptonemal complex (SC) formation. The deleted region included the entire $\mathrm{Xp}$ pseudoautosomal segment, and the most distal DNA sequences to have been mapped within it so far show a GC rich polymorphic minisatellite DXYS14 (probe 29A24) of six to 15 copies lying within only $25 \mathrm{kbp}$ of the telomere, with homologous copies at distal Yp. ${ }^{923}$ This unique observation prompted the question as to whether the GC rich repetitive sequences within the minisatellite $D X Y S 14$, and other GC rich minisatellites in the pseudoautosomal region, were in fact responsible for synaptic initiation in the $X Y$ pair. If so, was there circumstantial evidence that GC rich tandem repeats might function also in this way within the autosomal component of the human genome?

\section{Synapsis and crossing over in man}

Unlike the $\mathrm{XY}$ pair in which synapsis initiates at a single distal location, human autosomes can initiate their pairing at two ends and they also show interstitial points of synaptic initiation which are common in oocytes, but rarer in spermatocytes. ${ }^{1}$ The striking observation in both human oocytes and spermatocytes is, however, that pairing and SC formation most conspicuously begin at early zygotene in subtelomeric regions of all autosomes, and some time before interstitial stretches of the SC are seen (fig 1). The rich clustering of highly variable GC rich minisatellites also within human autosomal protermini ${ }^{6}$ is therefore of some significance, for it

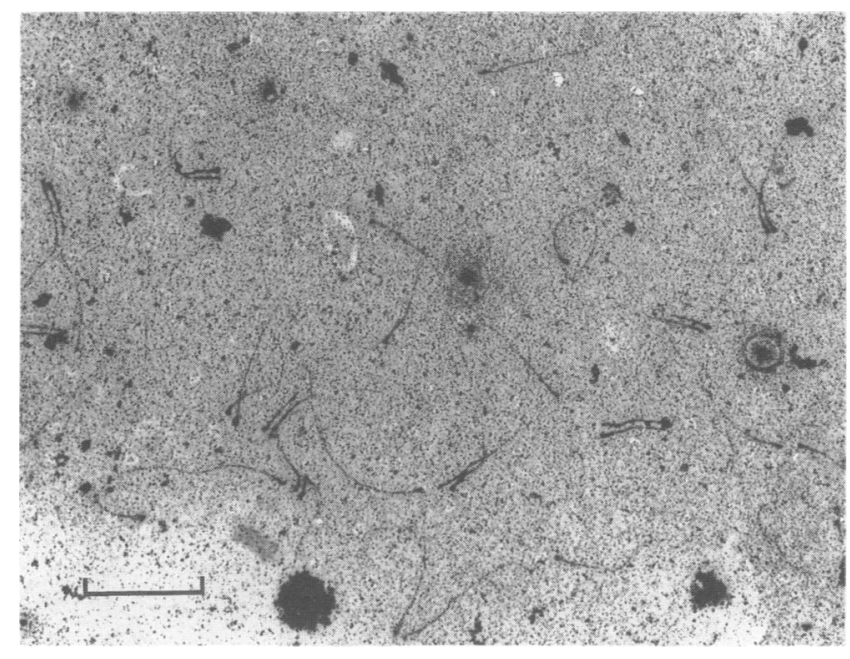

FIG 1 Electron microscope (EM) microspread preparation of an early zygotene human spermatocyte at the time of pairing initiation. Only within subtelomeric regions has formation of the SC started, interstitial segments still being only barely visible as single unpaired lateral elements. Bar $=10 \mu \mathrm{m}$. Silver nitrate stain. 
is very reminiscent of the situation found within the XY pair. ${ }^{910} 23$

The process of chromosome pairing in any eukaryote, from initiation at zygotene to desynapsis at diplotene, can readily be followed at electron microscope level by studying the behaviour of the SC, the tripartite proteinaceous structure which holds homologues together for recombination. ${ }^{1}$ The $\mathrm{SC}$ is obviously required for crossing over as little recombination takes place in its absence, ${ }^{24}$ but its presence alone does not guarantee crossing over, as the numerous published examples of illegitimate SC formation can testify. ${ }^{12526}$ Pairing at the SC level, in fact, seems to be an extremely flexible process and not necessarily indicative of homology.

Carpente ${ }^{27}$ has argued that synapsis can begin in any place where two unpaired lateral elements (LE) come close enough to one another. She sees the early zygotene stage as a time when a large number of transient synaptic trials takes place, non-homo- logous pairings within the SC occurring frequently until 'homology' checking is finished and stable homologous pairing is established. Gross examples of LE misalignments and non-homologous 'ectopic' pairings have in fact been observed in our own studies into normal human oocytes and spermatocytes examined at the early pachytene stage of meiotic prophase ${ }^{28}$ (fig 2), these originating in early zygotene at the time of pairing initiation and being about ten times more common in oocytes. ${ }^{28}$ The longer overall SC complement length in oocytes ${ }^{29}$ may perhaps be an important factor here, making them more error prone. Moreover, considerable variation between fetuses is found in the frequency of such errors ${ }^{28}$ indicating perhaps a certain 'genetic pairing instability' between different people. The clear presence of interchanges between non-homologues (translocations) at this time in meiotic prophase (fig $2 \mathrm{c}, \mathrm{d}$ ) indicates the previous occurrence of ectopic pairing and crossing over.
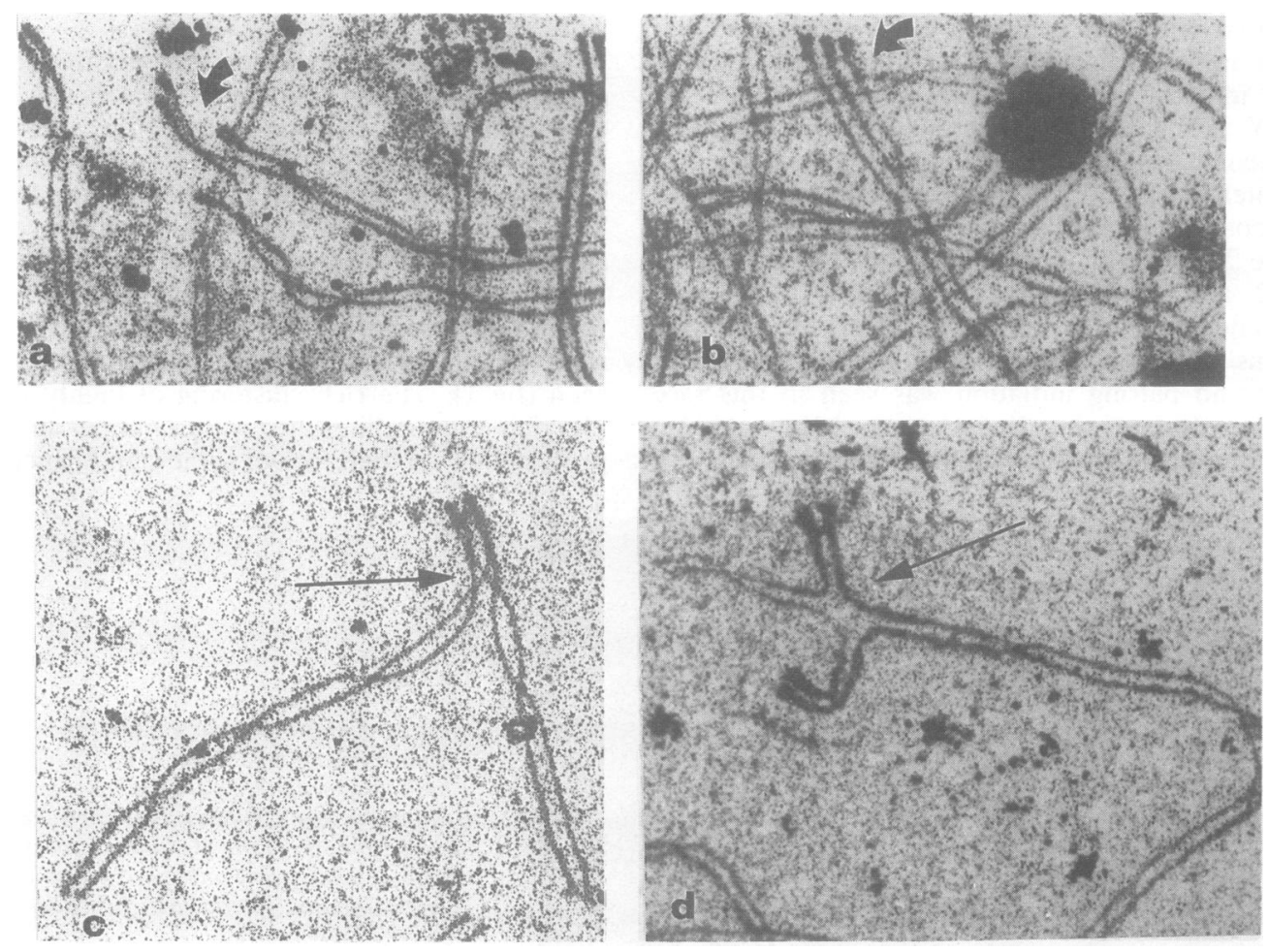

FIG 2 Human EM microspreads at the pachytene stage showing gross evidence of synaptic mismatch and misalignment in terminal segments (arrows). (a) Non-homologous partner switch and misalignment in an oocyte. (b) Triple association between one homologous SC and a single non-homologous lateral element in an oocyte. (c, d) Non-homologous interchange (translocation) after ectopic pairing in (c) spermatocyte, (d) oocyte. (Fig $2 a, b$, and d reproduced from Speed, ${ }^{28}$ with permission.) 


\section{Unequal crossing over within repeated sequences}

A vast number of published reports exists on unequal crossing over within repeated sequences of eukaryote genomes. ${ }^{16} 18$ In regions of tandem repeat, unequal crossing over can readily arise when there are two or more identical sites and hence substantial opportunity for misalignment between reiterated copies. Thus, the Bar mutation in Drosophila $^{30}$ is a direct tandem duplication of seven bands of the polytene map. Unequal crossing over results when the distal repeat of one chromosome recombines with the proximal repeat in the opposite homologue to yield $\mathrm{Bar}^{+}$revertants and double Bar progeny. Data from Drosophila also show how unequal crossing over can occur in portions of the genome where tandem duplication is not evident. After investigation into the molecular structure of reciprocal duplication/deficiency products of unequal crossing over in females heterozygous for various white $(w)$ alleles, Goldberg et al ${ }^{31}$ showed that asymmetrical pairing between staggered transposons (nomadic repetitive 'BEL' sequences) was responsible. Even very small $(8 \mathrm{~kb})$ displaced regions of homology within the BEL repeats were able to pair with one another with a considerable frequency, despite separation by $60 \mathrm{~kb}$ and despite being surrounded by extensive regions of standard homology. Other repetitive transposable elements such as 'copia' in Drosophila and Ty elements in yeast can also provide homology for unequal exchange. ${ }^{31}$ Tartof $^{18}$ estimates that the frequency of transposon mediated unequal exchange in Drosophila might be as high as $3 \times 10^{-3}$ per meiosis, a very high rate for a mutagenic process, and one which, he points out, has considerable implications for a species like man (see below).

\section{Pairing initiation within GC repeats and its consequences}

The preferential (though not exclusive) clustering of GC rich minisatellites in the protermini of human chromosomes, where meiotic pairing is seen principally to begin after telomeric association, is, I believe, strong circumstantial support for the belief that the two might be causally related. A recent in vitro study ${ }^{32}$ has shown that $G$ rich motifs in human DNA can, by special G-G bonding, form selfassociating structures (G4-DNA), a property not shared by AT rich sequences. Sen and Gilbert ${ }^{32}$ have suggested that G4-DNA might be responsible for meiotic pairing. Were $G$ rich motifs to be important in pairing, proterminal minisatellites with their high $G$ content core sequence would be powerful primary sites for synaptic initiation within terminal $\mathbf{R}$ bands. Moreover, their extreme variability might arise out of this early lead in pairing, since misalignment and unequal exchange owing to synaptic trial and error could be a common occurrence at the outset of the pairing process. $G$ rich interstitial minisatellites and short repetitive Alu sequences might also initiate pairing along arms (in interstitial $R$ bands), $R$ band regions opening up at the prophase of meiosis to facilitate the process. ${ }^{33}$ AT rich repeats may play a more passive role in pairing, being brought into alignment later when homology is becoming established. The finding of shorter length allelic changes in AT rich minisatellites in man $^{10}$ could be a reflection of this progression to homology. Other regional constraints on crossing over in AT rich regions (dark $G$ bands) might occur, however, attributable to a denser DNA conformation. ${ }^{34}$

By pachytene, it is envisaged that homology will be established and only reciprocal recombination will occur, a gradient of declining unequal exchange being expected between the zygotene and pachytene stages. The full machinery of chiasma formation ${ }^{35} 36$ will then be brought into play as a requirement for orderly segregation within bivalents. Many nonreciprocal exchanges (conversion type events) will not generate chiasmata, ${ }^{35}$ solving the apparent paradox of why recombination appears to occur almost anywhere in the genome of eukaryotes and yet chiasmata resulting from reciprocal crossing over are seen to be so clearly non-random in location. ${ }^{11}$ Conversions appear to be at least as common as reciprocal crossovers in eukaryotes but are not subject to interference. ${ }^{27}$ Their occurrence is quite independent of reciprocal crossing over and does not interfere with it in any way. ${ }^{27} 35$ Both initial alignment of chromosomes at zygotene and precise pairing at pachytene, together with their attendant crossover events, appear to be mediated by recombination nodules, ${ }^{27}$ and zytogene DNA synthesis, ${ }^{136}$ delayed from $\mathrm{S}$ phase, appears also to serve a role, although it is not yet clear what this might be. However, it is of some interest that zyg-DNA has a GC content of 50 to $53 \%,{ }^{1}$ similar to that found for Alu repeats. ${ }^{15}$

\section{Implications of the model for human genetic disease inheritance}

The idea of $G$ rich repetitive sequences functioning as pairing initiators at meiosis, with the inherent potential for unequal exchange after misalignment, has far reaching implications for man. Human genetics publications are replete with examples of de novo mutational change arising within the germ line, much of which could be the consequence of 


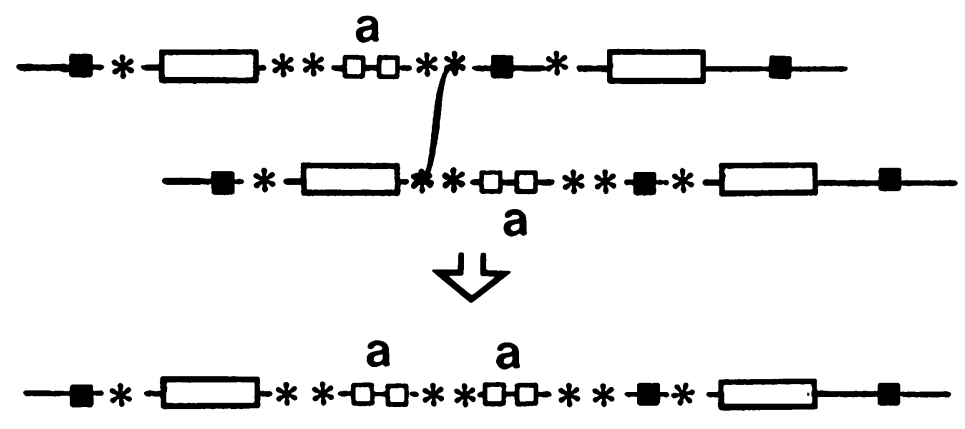

FIG 3 A hypothesised complex gene in which an unequal crossover between two misaligned repeat sequences $\left({ }^{* *}\right)$ has produced a duplication and deficiency for locus $a$.

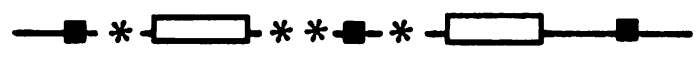

asymmetrical pairing and unequal exchange. In my view, such errors are likely to arise within repeat sequences at the time when an initial search for synaptic homology is being made at very early meiotic prophase. If short interspersed repeats (like Alu sequences) act to provide homology for pairing, an infinite variety of mutations, from minor gene conversions to duplications, deficiencies (fig 3 ), and inversions could arise, while ectopic pairing between heterologues could produce de novo translocations. Evidence for the occurrence of gene conversions in the human fetal globin ${ }^{37}$ and immunoglobulin ${ }^{38}$ genes exists, while molecular data show that unequal crossover events have occurred within Alu repeats giving duplication/deletion products at the hypercholesterolaemia ${ }^{39}$ locus and within the $\alpha$ globin cluster. ${ }^{40}$ Unequal exchanges within Alu sequences on Xp and Yp are documented in the origin of some $\mathrm{XX}$ males. ${ }^{41}$

In cancers, imprecise exchanges appear to have occurred within Alu repeats to produce the specific translocations associated with human chronic myeloid leukaemia $t(9 ; 22)^{42}$ and in murine plasmacytomas $\mathrm{T}(15 ; 12),{ }^{43}$ the latter being a translocation similar to that found in Burkitt's lymphoma $t(8 ; 14)$ in man. The evidence for unequal exchange in these sporadic tumours indicates that asymmetrical pairing between repetitive sequences on non-homologues not only occurs in the germ line but can also occur in somatic cells (although this is expected to be a much rarer event), and a first step by which potentially malignant cells establish homozygosity or hemizygosity for somatically recessive cancer genes ${ }^{192044}$ may be found in this process of mismatch.

The fragile $\mathrm{X}$ locus at $\mathrm{Xq} 27$ may be seen as a site resembling in its behaviour the Bar locus in Drosophila. Amplification within repetitive sequences at the common fragile site could produce the "premutation', ${ }^{45}$ with further amplification or deletion brought about by unequal crossing over giving rise to the full Martin-Bell syndrome. ${ }^{46}$ For human reciprocal translocations, imprecise exchange around breakpoints occurring in germ cells will be expected to give rise to associated congenital defect in offspring, and many known examples of mental and physical handicap associated with de novo translocation are in fact documented. ${ }^{47} \mathrm{~A}$ recent molecular investigation ${ }^{48}$ into the breakpoint sequences of an $X ; 21$ translocation in a girl with Duchenne muscular dystrophy shows $G$ rich motifs on both sides of a breakpoint with clear evidence that imprecise exchange has occurred during its formation. For balanced reciprocal translocations in a variety of species, including man, pairing is frequently disturbed around breakpoints ${ }^{4-52}$ with resulting crossover suppression. ${ }^{49}$ This could arise out of disruption caused in what normally would be a pairing site at meiosis.

For proterminal GC rich minisatellites in man, the emphasis in past publications has always been on their 'recombinational' proficiency. ${ }^{3-6}$ In my view, the cart has been put before the horse; for it is their 'pairing' proficiency which I believe should be emphasised first and foremost. Without pairing there can be no recombination, and if $G$ rich repetitive sequences initiate synapsis, there is likely to be maximum opportunity in GC rich minisatellites for mismatch and unequal exchange. Moreover, those same minisatellites which show instability in the germ line might be expected to show similar characteristics in somatic tissues, and in normal as well as cancer cells, a prediction which appears to hold true. ${ }^{53}$

Far from believing that simple repeats interspersed around eukaryotic genomes may constitute 'junk' DNA and be lacking in function, ${ }^{54}$ I believe their role in synapsis to be one of major importance and one which has been largely overlooked by molecular 
investigators. Perhaps human gene mapping should concentrate its efforts on hot spots of recombination throughout the entire genome and study in detail the DNA sequences existing at those sites, as Siniscalco ${ }^{17}$ has previously suggested. This could lead to a fuller understanding of how a specific mutant phenotype might have arisen out of an unequal exchange event within any particular gene locus.

The author wishes to acknowledge the expertise of her colleague $\mathbf{R}$ M Speed in preparing and observing human meiotic prophase spreads. She is also indebted to Dr Veronica Buckle for many helpful discussions and for her encouragement and support during the preparation of the paper. Professor Marcus Pembrey gave advice on the inheritance of the fragile $X$ syndrome and is thanked for his help. Professor J M Connor is also thanked for his encouragement and support. The manuscript was kindly typed by Mrs Ann Kenmure. Professor H J Evans is thanked for his comments.

\section{References}

1 von Wettstein D, Rasmussen SW, Holm PB. The synaptonemal complex in genetic segregation. Annu Rev Genet 1984;18: 331-413.

2 Singer MF. Highly repeated sequences in mammalian genomes. Int Rev Cytol 1982;76:67-112.

3 Jeffreys AJ, Wilson V, Thein SL. Hypervariable 'minisatellite regions in human DNA. Nature 1985;314:67-73.

4 Jeffreys AJ, Royle NJ, Wilson V, Wong Z. Spontaneous mutation rates to new length alleles at tandem repetitive hypervariable loci in human DNA. Nature 1987;332:278-81.

5 Nakamura Y, Leppert M, O'Connell P, Wolff R, et al. Variable number of tandem repeat (VNTR) markers for human gene mapping. Science 1987;235:1616-22.

6 Jarman AP, Higgs DR. A new hypervariable marker for the human $\alpha$-globin gene cluster. Am J Hum Genet 1988;43:249-56.

7 Royle NJ, Clarkson RE, Wong Z, Jeffreys AJ. Clustering of hypervariable minisatellites in the proterminal regions of human autosomes. Genomics 1988;3:352-60.

${ }^{8}$ Oberlé I, Drayna D, Camerino G, White R, Mandel J-L. The telomeric region of the human $X$ chromosome long arm: presence of a highly polymorphic DNA marker and analysis of recombination frequency. Proc Natl Acad Sci USA 1985;82: 2824-8.

9 Cooke HJ, Brown WRA, Rappold GA. Hypervariable telomeric sequences from the human sex chromosomes are pseudoautosomal. Nature 1985;317:687-92.

10 Simmier M-C, Johnsson C, Petit C, Rouyer F, Vergnaud G, Weissenbach $\mathrm{J}$. Two highly polymorphic minisatellites from the pseudoautosomal region of the human sex chromosomes. EMBO J 1987;6:963-9.

1 Hultén MA. Chiasma distribution at diakinesis in the normal human male. Hereditas 1974;76:55-78.

12 Rouyer F, Simmler MC, Johnsson C, Vergnaud G, Cooke HJ, Weissenbach J. A gradient of sex linkage in the pseudoautosomal region of the human sex chromosomes. Nature 1986;319: 291-5.

13 Jeffreys AJ, Morton DB. DNA fingerprints of dogs and cats. Anim Genet 1987;18:1-15.

14 Rogstad SH, Patton JC, Schaal BA. M13 repeat probe detects DNA minisatellite-like sequences in gymnosperms and angiosperms. Proc Natl Acad Sci USA 1988;85:9176-8.

15 Korenberg JR, Rykowski MC. Human genome organisation:
$\mathrm{Alu}$, Lines and the molecular structure of metaphase chromosome bands. Cell 1988;53:391-400.

16 Smith GP. Evolution of repeated DNA sequences by unequal crossover. Science 1976;191:528-35.

17 Siniscalco M. Genetic recombination and disease. Cold Spring Harbor Symp Quant Biol 1986;51:191-4.

18 Tartof KD. Unequal crossing over then and now. Genetics 1988;120:1-6.

19 Bishop JM. The molecular genetics of cancer. Science 1987;235: 305-11.

20 Yunis JJ. The chromosomal basis of human neoplasia. Science 1983;221:227-36.

21 Hall JG. Review and hypothesis. Somatic mosaicism: observations related to clinical genetics. Am J Hum Genet 1988;43: 355-63.

22 Chandley AC, Goetz P, Hargreave TB, Joseph AM, Speed RM. On the nature and extent of $\mathrm{XY}$ pairing at meiotic prophase in man. Cytogenet Cell Genet 1984;38:241-7.

23 Cooke HJ, Smith BA. Variability at the telomeres of the human $\mathrm{X} / \mathrm{Y}$ pseudoautosomal region. Cold Spring Harbor Symp Quant Biol 1986;51:213-9.

24 Carpenter ATC. Electron microscopy of meiosis in Drosophila melanogaster females. I. Structure, arrangement, and temporal change of the synaptonemal complex in wild-type. Chromosoma 1975;51:157-82.

25 Moses MJ. Microspreading and the synaptonemal complex in cytogenetic studies. In: de la Chapelle A, Sorsa M, eds. Chromosomes today. Vol 6. Amsterdam: Elsevier/North Holland Biomedical Press, 1977: 71-82.

26 Gillies CB. The synaptonemal complex in higher plants. Crit Rev Plant Sci 1984:2:81-116.

27 Carpenter ATC. Gene conversion, recombination nodules and the initiation of meiotic synapsis. Bio Essays 1987;6:232-6.

28 Speed RM. The possible role of meiotic pairing anomalies in the atresia of human fetal oocytes. Hum Genet 1988;78:260-6.

29 Bojko M. Human meiosis IX. Crossing over and chiasma formation in oocytes. Carlsberg Res Commun 1985;50:43-72.

30 Sturtevant AH. The effects of unequal crossing over at the bar locus in Drosophila. Genetics 1925;10:117-47.

31 Goldberg ML, Sheen J-Y, Gehring WJ, Green MM. Unequal crossing-over associated with asymmetrical synapsis between nomadic elements in the Drosophila melanogaster genome. Proc Natl Acad Sci USA 1983;80:5017-21.

32 Sen D, Gilbert W. Formation of parallel four-stranded complexes by guanine-rich motifs in DNA and its implications for meiosis. Nature 1988;334:364-6.

${ }^{33}$ Luciani JM, Guichaoua MR, Cau P, Devictor B, Salagnon N. Differential elongation of autosomal pachytene bivalents related to their DNA content in human spermatocytes. Chromosoma 1988;97:19-25.

34 Holmquist GP. Role of replication time in the control of tissuespecific gene expression. Am J Hum Genet 1987;40:151-73.

35 Holliday R. Recombination and meiosis. Philos Trans $R$ Soc Lond [Biol] 1977;277:359-70.

36 Stern H, Hotta Y. Biochemistry of meiosis. Philos Trans $R$ Soc Lond [Biol] 1977;277:277-94.

37 Smithies O, Powers PA. Gene conversions and their relation to homologous chromosome pairing. Philos Trans $R$ Soc Lond [Biol] 1986;312:291-302.

38 Maizels N. Might gene conversion be the mechanism of somatic hypermutation of mammalian immunoglobulin genes? Trends Genet 1989;5:4-8.

39 Lehrman MA, Goldstein JL, Russell DW, Brown MS. Duplication of seven exons in LDL receptor gene caused by Alu-Alu recombination in a subject with familial hypercholesterolemia. Cell 1987;48:827-35.

40 Nicholls RD, Fischel-Ghodsian N, Higgs DR. Recombination at the human $\alpha$-globin gene cluster: sequence features and topological constraints. Cell 1987;49:369-78.

41 Rouyer F, Simmler M-C, Page DC, Weissenbach J. A sex 
chromosome rearrangement in a human XX male caused by ALU-ALU recombination. Cell 1987;51:417-25.

42 de Klein A, van Agthoven T, Groffen C, Heister Kamp N, Groffen J, Grosveld G. Molecular analysis of both translocation products of a Philadelphia-positive CML patient. Nucleic Acids Res 1986;14:7071-82.

${ }^{43}$ Gerondakis S, Cory S, Adams JM. Translocation of the myc cellular oncogene to the immunoglobulin heavy chain locus in murine plasmacytomas is an imprecise reciprocal exchange. Cell 1984;36:973-82.

44 Knudson AG. Mutation and cancer: statistical study of retinoblastoma. Proc Natl Acad Sci USA 1971;68:820-3.

45 Pembrey M, Winter RM, Davies KE. A premutation that generates a defect at crossing-over explains the inheritance of fragile X mental retardation. Am J Med Genet 1985;21:709-17.

46 Nussbaum RL, Leadbetter DH. Fragile $X$ syndrome: a unique mutation in man. Annu Rev Genet 1986;20:109-45.

47 Jacobs PA. Correlation between euploid structural chromosome rearrangements and mental subnormality in humans. Nature 1974;249:164-5.

48 Bodrug SE, Ray PN, Gonzalez IL, Schmickel RD, Sylvester JE, Worton RG. Molecular analysis of a constitutional X-autosome translocation in a female with muscular dystrophy. Science 1987;237:1620-4.
49 Roberts PA. Screening for X-ray induced crossover suppressors in Drosophila melanogaster: prevalence and effectiveness of translocations. Genetics 1970;65:429-48.

50 Gabriel-Robez O, Jaafar H, Ratomponirina C, Boscher J. Heterosynapsis in a heterozygous fertile boar carrier of a $3 ; 7$ translocation. Chromosoma 1988;97:26-32.

51 Chandley AC, Speed RM, McBeath S, Hargreave TB. A human 9;20 reciprocal translocation associated with male infertility analyzed at prophase and metaphase 1 of meiosis. Cytogenet Cell Genet 1986;41:145-53.

52 Gillies GB. Electron microscopy of spread maize pachytene synaptonemal complexes. Chromosoma 1981;83:575-91.

53 Armour JAL, Patel I, Thein SL, Fey MF, Jeffreys AJ. Analysis of somatic mutations at human minisatellite loci in tumours and cell lines. Genomics 1989;4:328-34.

54 Doolittle WF, Sapienza C. Selfish genes, the phenotype paradigm and genome evolution. Nature 1980;284:601-3.

Correspondence to Dr A C Chandley, MRC Human Genetics Unit, Western General Hospital, Crewe Road, Edinburgh EH4 2XU. 